\title{
Studies of Phosphazenes. Part 30.' Reactions of Hexachlorocyclotriphosphazene with Aromatic Primary Amines: Interplay of Geminal and Non-geminal Modes of Chlorine Replacement $\dagger$
}

\author{
Sivapackia Ganapathiappan and Setharampattu S. Krishnamurthy* \\ Department of Inorganic and Physical Chemistry, Indian Institute of Science, Bangalore 560012 , India
}

The reactions of hexachlorocyclotriphosphazene, $\mathrm{N}_{3} \mathrm{P}_{3} \mathrm{Cl}_{6}$, with aromatic primary amines (aniline, $p$-toluidine, and $p$-anisidine) yield the derivatives $\mathrm{N}_{3} \mathrm{P}_{3} \mathrm{Cl}_{6-n}\left(\mathrm{NHC}_{6} \mathrm{H}_{4} \mathrm{Y}-p\right)_{n}[\mathrm{Y}=\mathrm{H}, \mathrm{Me}$, or OMe, $n=1$ or 2 (three isomers); $\mathrm{Y}=\mathrm{OMe}, n=3$ (three isomers), 4 (geminal isomer only), 5, or 6 ]. The structures of these derivatives and their isomeric compositions have been determined by ${ }^{31} \mathrm{P}$ n.m.r. spectroscopy and also by ${ }^{1} \mathrm{H}$ n.m.r. spectra of the corresponding methoxy and/or dimethylamino derivatives. When diethyl ether, tetrahydrofuran, or $\mathrm{MeCN}$ is used as the solvent, non-geminal products predominate at the bis stage of chlorine replacement. However, in the presence of triethylamine, the geminal product is formed exclusively. At the tetrakis stage of chlorine replacement, the geminal isomer is the sole product irrespective of the reaction medium. The reaction of non-gem- $\mathrm{N}_{3} \mathrm{P}_{3} \mathrm{Cl}_{3}\left[\mathrm{NHC}_{6} \mathrm{H}_{4}(\mathrm{OMe})-p\right]_{3}$ with $p$-anisidine yields only $\mathrm{N}_{3} \mathrm{P}_{3}\left[\mathrm{NHC}_{6} \mathrm{H}_{4}(\mathrm{OMe})-p\right]_{6}$; formation of non-geminal tetrakis derivatives could not be detected. The mechanistic implications of these results are discussed.

In our investigation of the reactions of hexachlorocyclotriphosphazene, $\mathrm{N}_{3} \mathrm{P}_{3} \mathrm{Cl}_{6} \quad(1), \ddagger$ with aromatic diamines (benzidine and 4,4'-oxydianiline) with a view to synthesizing phosphazene-based polymers, we have found that these reactions in tetrahydrofuran (thf) (with or without triethylamine) yield an extremely complex mixture of products which are not amenable to separation by chromatographic or fractional crystallization techniques. In order to assess the reactivities of these diamines towards (1), we have carried out kinetic studies of the reactions of (1) with aromatic primary amines (aniline, $p$-toluidine, and $p$-anisidine) as 'model systems' for the phosphazene-based polymers and observed that the second stage of chlorine replacement in methyl cyanide (MeCN) proceeds by a bimolecular mechanism. ${ }^{2}$ This result implies that the products formed are presumably the non-geminal bis(amino) derivatives in contrast to the previous report of a predominantly geminal mode of substitution established for aniline in benzene. ${ }^{3.4}$ Therefore, a detailed study of the products formed in the reactions of (1) with aromatic primary amines under various experimental conditions has been undertaken and the results are presented in this paper. The results of the kinetic studies are reported in the following paper. ${ }^{2}$

\section{Experimental}

Hexachlorocyclotriphosphazene, $\mathrm{N}_{3} \mathrm{P}_{3} \mathrm{Cl}_{6}$ (1), was prepared and purified by a standard method. ${ }^{5}$ The solvents were purified by conventional procedures. Light petroleum employed had a boiling point range of $60-80^{\circ} \mathrm{C}$. Triethylamine was distilled and stored over potassium hydroxide pellets. Aniline (B.D.H.) was distilled over zinc dust in vacuo. p-Toluidine (B.D.H.) was recrystallized from $85 \%$ ethanol and dried thoroughly before use. $p$-Anisidine (Sigma Chemicals) was sublimed $\left(80^{\circ} \mathrm{C} / 0.1\right.$ Torr, $\approx 13 \mathrm{~Pa}$ ) and used within $24 \mathrm{~h}$.

The reactions were carried out by varying the stoicheiometry

$\dagger$ Supplementary data available (No. SUP 56656, 4 pp.): analytical data. See Instructions for Authors, J. Chem. Soc., Dalton Trans., 1987, Issue 1, pp. $x$ vii- $x \mathbf{x}$.

$\ddagger$ A more systematic name for this compound is $2,2,4,4,6,6$-hexachlorocyclotri( $\lambda^{5}$-phosphazene $)$ of the reactants and the solvent. The general procedure is described below and details of the experiments and the products obtained are summarized in Table 1 .

General Procedure.-The aromatic primary amine, dissolved in an appropriate solvent, was added to a solution of (1) with or without triethylamine in the same solvent over a period of $1 \mathrm{~h}$ at ambient temperature $\left(\mathrm{ca} .25^{\circ} \mathrm{C}\right)$. The course and the extent of the reaction were monitored by t.l.c. The reaction mixture was stirred at ambient temperature or heated under reflux till the consumption of the reacting amine was complete. The amine hydrochloride was filtered off and the solvent from the filtrate was evaporated under reduced pressure. The products were separated by fractional crystallization (1:1 light petroleumdichloromethane) and/or by column chromatography [benzene or benzene-ethyl acetate $(10: 1)$ as eluant].

The chloro precursors were characterized further by converting them into their dimethylamino and/or methoxy derivatives. Dimethylaminolysis was carried out in boiling chloroform and methoxylation in boiling thf. Complete chlorine replacement was achieved for all the compounds except for (17). For the latter compound, more forcing conditions were required and hence the reaction was carried out in boiling $\mathrm{MeCN}$. In general the yields of the dimethylaminated derivatives were $>95 \%$ and those of the methoxylated derivatives $60-85 \%$.

The melting points of the compounds were determined using a Reichert-Kofler micro-heating stage fitted with a polarizing microscope and are uncorrected. Details of n.m.r. and i.r. spectroscopic measurements are described elsewhere. ${ }^{6}$ I.r. spectra of (arylamino)cyclotriphosphazenes show bands in the ranges $1160-1220,3200-3420$, and $450-640 \mathrm{~cm}^{-1}$ attributable to $v(\mathrm{P}=\mathrm{N}), v(\mathrm{~N}-\mathrm{H})$, and $v(\mathrm{P}-\mathrm{Cl})$ respectively. Satisfactory elemental analyses were obtained for all the new compounds.

\section{Results and Discussion}

Preparative and Spectroscopic Aspects.-The nature of the products formed up to the bis stage of chlorine replacement is the same for all three aromatic primary amines. At the second stage of chlorine replacement, a non-geminal derivative is formed predominantly in diethyl ether, thf, or $\mathrm{MeCN}$. If 
Table 1. Experimental details for the reactions of $\mathrm{N}_{3} \mathrm{P}_{3} \mathrm{Cl}_{6}$ (1) with aniline, $p$-toluidine, and $p$-anisidine ${ }^{a}$

\begin{tabular}{|c|c|c|c|c|c|c|}
\hline \multirow[b]{2}{*}{$\begin{array}{l}\text { Amine } \\
(\mathrm{mmol})\end{array}$} & \multirow{2}{*}{$\begin{array}{l}\text { Solvent } \\
\text { (total } \\
\text { volume/ } \\
\mathrm{cm}^{3} \text { ) }\end{array}$} & \multirow{2}{*}{$\begin{array}{c}\text { Total } \\
\text { reaction } \\
\text { time } / \\
\mathrm{h}\end{array}$} & \multicolumn{4}{|c|}{ Products and yields } \\
\hline & & & Compound(s) & $\begin{array}{c}R_{\mathrm{f}} \\
\text { values }^{b}\end{array}$ & $\begin{array}{l}\text { Amount/ } \\
\mathrm{g}\end{array}$ & $\begin{array}{l}\text { Yield }^{c} / \\
\%\end{array}$ \\
\hline $\begin{array}{l}\text { Aniline } \\
(20)\end{array}$ & $\begin{array}{c}\mathrm{MeCN} \\
(150)\end{array}$ & 48 & $\begin{array}{c}(2) \\
(3)+(4)\end{array}$ & $\begin{array}{l}0.73^{d} \\
0.47^{d}\end{array}$ & $\begin{array}{c}3.60 \\
\text { Trace }\end{array}$ & $\begin{array}{c}90.0 \\
e\end{array}$ \\
\hline $\begin{array}{l}\text { Aniline } \\
\text { (40) }\end{array}$ & $\begin{array}{l}\mathrm{Et}_{2} \mathrm{O} \\
(150)\end{array}$ & 360 & $\begin{array}{l}\text { (2) } \\
\text { (3) }+(5)\end{array}$ & $\begin{array}{l}0.73^{d} \\
0.47^{d} \\
0.47 \\
0.14^{d}\end{array}$ & $\begin{array}{c}2.40 \\
0.38 \\
\text { Trace }\end{array}$ & $\begin{array}{c}59.3 \\
8.3 \\
e\end{array}$ \\
\hline $\begin{array}{l}\text { Aniline }^{f} \\
\text { (20) }\end{array}$ & $\begin{array}{l}\text { thf } \\
(150)\end{array}$ & 24 & (3) & $0.47^{d}$ & 4.40 & 95.0 \\
\hline $\begin{array}{l}\text { Aniline } \\
\text { (40) }\end{array}$ & $\begin{array}{c}\mathrm{MeCN} \\
(200)\end{array}$ & 192 & $\begin{array}{c}(2) \\
(4) \\
(5)+(6)\end{array}$ & $\begin{array}{l}0.73^{d} \\
0.47^{d} \\
0.14^{d}\end{array}$ & $\begin{array}{l}\text { Trace } \\
2.64 \\
\text { Trace }\end{array}$ & $\begin{array}{c}e \\
68.2 \\
e\end{array}$ \\
\hline $\begin{array}{l}p \text {-Toluidine } \\
\text { (20) }\end{array}$ & $\begin{array}{c}\mathrm{MeCN} \\
(200)\end{array}$ & 36 & $\begin{array}{c}(7) \\
(8)+(9)\end{array}$ & $\begin{array}{l}0.79^{d} \\
0.57^{d}\end{array}$ & $\begin{array}{l}3.50 \\
\text { Trace }\end{array}$ & $\begin{array}{c}83.7 \\
e\end{array}$ \\
\hline $\begin{array}{l}p \text {-Toluidine } \\
\text { (40) }\end{array}$ & $\begin{array}{l}\mathrm{Et}_{2} \mathrm{O} \\
(200)\end{array}$ & 360 & $\begin{array}{l}(7) \\
(9) \\
(8)+(10)\end{array}$ & $\begin{array}{l}0.79^{d} \\
0.57^{d} \\
0.57^{d} \\
0.32^{d}\end{array}$ & $\begin{array}{c}2.50 \\
0.52 \\
\text { Trace }\end{array}$ & $\begin{array}{c}59.8 \\
10.6 \\
e\end{array}$ \\
\hline $\begin{array}{l}p \text {-Toluidine } \\
\text { (40) }\end{array}$ & $\begin{array}{l}\mathrm{MeCN} \\
(200)\end{array}$ & 192 & $\begin{array}{c}(7) \\
(9) \\
(10)+(11)\end{array}$ & $\begin{array}{l}0.79^{d} \\
0.57^{d} \\
0.32^{d}\end{array}$ & $\begin{array}{l}\text { Trace } \\
2.77 \\
\text { Trace }\end{array}$ & $\begin{array}{c}e \\
56.8 \\
e\end{array}$ \\
\hline $\begin{array}{l}p \text {-Toluidine }{ }^{f} \\
\quad(20)\end{array}$ & $\begin{array}{l}\text { thf } \\
(150)\end{array}$ & 24 & (8) & $0.57^{d}$ & 4.60 & 94.0 \\
\hline $\begin{array}{l}p \text {-Anisidine } \\
(20)\end{array}$ & $\begin{array}{l}\text { MeCN } \\
(200)\end{array}$ & 36 & $\begin{array}{c}(12) \\
(13)+(14)\end{array}$ & $\begin{array}{l}0.57^{d} \\
0.40 \\
0.33^{d}\end{array}$ & $\begin{array}{l}3.45 \\
\text { Trace }\end{array}$ & $\begin{array}{c}80.0 \\
e\end{array}$ \\
\hline $\begin{array}{l}\text { p-Anisidine } \\
\text { (40) }\end{array}$ & $\begin{array}{l}\mathrm{Et}_{2} \mathrm{O} \\
(125)\end{array}$ & 360 & $\begin{array}{c}(12) \\
(14) \\
(13)+(15)\end{array}$ & $\begin{array}{l}0.57^{d} \\
0.33^{d} \\
0.40 \\
0.42^{d}\end{array}$ & $\begin{array}{c}0.43 \\
2.86 \\
\text { Trace }\end{array}$ & $\begin{array}{c}10.0 \\
55.0 \\
e\end{array}$ \\
\hline $\begin{array}{l}p \text {-Anisidine } \\
\text { (40) }\end{array}$ & $\begin{array}{l}\mathrm{MeCN} \\
(200)\end{array}$ & 192 & $\begin{array}{c}(12) \\
(14) \\
(13)+(15)+ \\
(16)\end{array}$ & $\begin{array}{l}0.57^{d} \\
0.33^{d} \\
0.40 \\
0.42 \\
0.58^{d}\end{array}$ & $\begin{array}{c}\text { Trace } \\
3.87 \\
\text { Trace }\end{array}$ & $\begin{array}{c}e \\
89.2 \\
e\end{array}$ \\
\hline $\begin{array}{l}p \text {-Anisidine } \\
\quad(60)\end{array}$ & $\begin{array}{c}\mathrm{MeCN} \\
(150)\end{array}$ & $40^{g}$ & $\begin{array}{c}(13) \\
(14) \\
(16) \\
(15)+(16)\end{array}$ & $\begin{array}{l}0.40^{d} \\
0.33^{d} \\
0.58 \\
0.42 \\
0.58\end{array}$ & $\begin{array}{l}0.20 \\
0.56 \\
0.18 \\
2.13\end{array}$ & $\begin{array}{r}3.6 \\
10.7 \\
3.5 \\
35.0\end{array}$ \\
\hline & & & $\begin{array}{l}(\mathbf{1 7}) \\
(\mathbf{1 9})\end{array}$ & $\begin{array}{l}0.23 \\
0.05\end{array}$ & $\begin{array}{l}0.27 \\
0.10\end{array}$ & $\begin{array}{r}33.9 \\
1.3\end{array}$ \\
\hline $\begin{array}{l}p \text {-Anisidine } \\
\quad(80)\end{array}$ & $\begin{array}{c}\mathrm{MeCN} \\
(150)\end{array}$ & $45^{g}$ & $\begin{array}{c}(13) \\
(14)+(16)\end{array}$ & $\begin{array}{l}0.40^{d} \\
0.33^{d} \\
0.42 \\
0.58\end{array}$ & $\begin{array}{l}0.03 \\
0.04 \\
0.80\end{array}$ & $\begin{array}{r}0.6 \\
0.8 \\
15.4\end{array}$ \\
\hline & & & $\begin{array}{l}(17) \\
(17)+(18)+ \\
(19)^{h}\end{array}$ & $\begin{array}{l}0.23 \\
0.23 \\
0.14 \\
0.05\end{array}$ & $\begin{array}{l}1.08 \\
0.08\end{array}$ & $\begin{array}{r}16.0 \\
1.2\end{array}$ \\
\hline $\begin{array}{l}p \text {-Anisidine } \\
(200)\end{array}$ & $\begin{array}{c}\mathrm{MeCN} \\
(150)\end{array}$ & $36^{g}$ & $\begin{array}{l}(19) \\
(19)\end{array}$ & $\begin{array}{l}0.05 \\
0.05\end{array}$ & $\begin{array}{l}1.45 \\
5.98\end{array}$ & $\begin{array}{l}18.5 \\
86.0\end{array}$ \\
\hline $\begin{array}{l}p \text {-Anisidine } \\
\text { (20) }\end{array}$ & $\begin{array}{l}\text { thf } \\
(150)\end{array}$ & $36^{g}$ & $\begin{array}{l}(13) \\
(17)\end{array}$ & $\begin{array}{l}0.40^{d} \\
0.23\end{array}$ & $\begin{array}{l}3.30 \\
0.40\end{array}$ & $\begin{array}{r}63.4 \\
5.8\end{array}$ \\
\hline $\begin{array}{l}p \text {-Anisidine } \\
\quad(30)\end{array}$ & $\begin{array}{l}\text { thf } \\
(150)\end{array}$ & $24^{g}$ & $\begin{array}{l}(13) \\
(17) \\
(19)\end{array}$ & $\begin{array}{l}0.40 \\
0.23 \\
0.05\end{array}$ & $\begin{array}{c}2.23 \\
2.68 \\
\text { Trace }\end{array}$ & $\begin{array}{c}42.8 \\
38.6 \\
e\end{array}$ \\
\hline $\begin{array}{l}p \text {-Anisidine }{ }^{f} \\
\quad(40)\end{array}$ & $\begin{array}{l}\text { thf } \\
(150)\end{array}$ & $42^{g}$ & $\begin{array}{l}\text { (13) } \\
(17) \\
(19)\end{array}$ & $\begin{array}{l}0.40^{d} \\
0.23 \\
0.05\end{array}$ & $\begin{array}{l}\text { Trace } \\
4.20 \\
\text { Trace }\end{array}$ & $\begin{array}{c}e \\
60.5 \\
e\end{array}$ \\
\hline
\end{tabular}

${ }^{a}$ In all the reactions, $\mathrm{N}_{3} \mathrm{P}_{3} \mathrm{Cl}_{6}(3.48 \mathrm{~g}, 10 \mathrm{mmol})$ was used. Reactions were carried out at $c a .25^{\circ} \mathrm{C}$ unless otherwise stated. See Table 2 for compound formulae. ${ }^{b}$ Eluant is ethyl acetate-benzene $(1: 10)$ unless otherwise stated. ${ }^{c}$ Reported yield of the products is after column chromatographic separation. ${ }^{d}$ Eluant is benzene. ${ }^{e}$ Not isolated; t.l.c. and spectroscopic evidence. ${ }^{\int}$ Triethylamine $\left(8.16 \mathrm{~cm}^{3}, 60 \mathrm{mmol}\right)$ was also added to $\mathrm{N}_{3} \mathrm{P}_{3} \mathrm{Cl}_{6}$. ${ }^{g}$ Reaction was carried out at the boiling point of the solvent. ${ }^{h}$ Contains equal amounts of (17), (18), and (19). Analytical data for this fraction [Found: $\mathrm{C}, 54.2 ; \mathrm{H}, 4.9 ; \mathrm{N}, 14.4 . \mathrm{C}_{35} \mathrm{H}_{40} \mathrm{ClN}_{8} \mathrm{O}_{5} \mathrm{P}_{3}(18)$ requires $\mathrm{C}, 53.8 ; \mathrm{H}, 5.2 ; \mathrm{N}, 14.4 \%$ ]. 
Table 2. Phosphorus-31 n.m.r. data for $\mathrm{N}_{3} \mathrm{P}_{3} \mathrm{Cl}_{6-n}(\mathrm{NHR})_{n}$ and their methoxy/dimethylamino derivatives ${ }^{a}$

\begin{tabular}{|c|c|c|c|c|c|}
\hline Compound $^{b}$ & M.p. $/{ }^{\circ} \mathrm{C}$ & $\delta\left(P^{1}\right)^{c}$ & $\delta\left(\mathrm{P}^{2}\right)^{d}$ & $\delta\left(\mathrm{P}^{3}\right)^{e}$ & ${ }^{2} J(\mathrm{PNP}) / \mathrm{Hz}$ \\
\hline (1) $\mathrm{N}_{3} \mathrm{P}_{3} \mathrm{Cl}_{6}$ & $113-114$ & 19.3 & & & \\
\hline $\begin{array}{l}\text { (2) } \mathrm{N}_{3} \mathrm{P}_{3} \mathrm{Cl}_{5}\left(\mathrm{NHR}^{1}\right) \\
\text { (3) gem- }-\mathrm{N}_{3} \mathrm{P}_{3} \mathrm{Cl}_{4}\left(\mathrm{NHR}^{1}\right)_{2}\end{array}$ & $98^{f}$ & 21.2 & 11.7 & & 47.1 \\
\hline $\begin{array}{l}\text { (3) gem- } \mathrm{N}_{3} \mathrm{P}_{3} \mathrm{Cl}_{4}\left(\mathrm{NHR}^{1}\right)_{2} \\
\text { (4) non-gem- } \mathrm{N}_{3} \mathrm{P}_{3} \mathrm{Cl}_{4}\left(\mathrm{NHR}^{1}\right)_{2}\end{array}$ & $\begin{array}{c}209^{g} \\
125-142^{h}\end{array}$ & 20.5 & & -2.7 & 48.2 \\
\hline $\operatorname{cis-(4)}{ }^{i}$ & & 22.3 & 13.8 & & 49.4 \\
\hline trans-(4) ${ }^{i}$ & & 22.2 & 13.5 & & 49.0 \\
\hline $\begin{array}{l}\text { (5) gem- } \mathrm{N}_{3} \mathrm{P}_{3} \mathrm{Cl}_{3}\left(\mathrm{NHR}^{1}\right)_{3} \\
\text { (6) non-gem- } \mathrm{N}_{3} \mathrm{P}_{3} \mathrm{Cl}_{3}\left(\mathrm{NHR}^{1}\right)_{3}{ }^{k}\end{array}$ & & & $j$ & & \\
\hline$c i s-(6)^{i}$ & & & 15.5 & & \\
\hline${\text { trans }-(6)^{i}}^{2}$ & & & 15.3 & & \\
\hline $\begin{array}{l}\text { (7) } \mathrm{N}_{3} \mathrm{P}_{3} \mathrm{Cl}_{5}\left(\mathrm{NHR}^{2}\right) \\
\text { (8) gem- } \mathrm{N}_{3} \mathrm{P}_{3} \mathrm{Cl}_{2}\left(\mathrm{NHR}^{2}\right)\end{array}$ & Viscous oil & 21.6 & 12.2 & & 48.2 \\
\hline $\begin{array}{l}\text { (8) gem- } \mathrm{N}_{3} \mathrm{P}_{3} \mathrm{Cl}_{4}\left(\mathrm{NHR}^{2}\right)_{2} \\
\text { (9) non-gem- } \mathrm{N}_{3} \mathrm{P}_{3} \mathrm{Cl}_{4}\left(\mathrm{NHR}^{2}\right)_{2}\end{array}$ & $\begin{array}{c}138^{\prime} \\
107-129\end{array}$ & 21.8 & & -1.6 & 48.5 \\
\hline $\begin{array}{l}\text { (9) non-gem- } \mathrm{N}_{3} \mathrm{P}_{3} \mathrm{Cl}_{4}\left(\mathrm{~N} n \mathrm{~N}-\mathrm{T}_{2}\right. \\
\text { cis-(9) }\end{array}$ & & 22.5 & 14.0 & & 49.6 \\
\hline trans $-(9)^{i}$ & & 22.3 & 13.9 & & 49.6 \\
\hline $\begin{array}{l}\text { (10) gem- } \mathrm{N}_{3} \mathrm{P}_{3} \mathrm{Cl}_{3}\left(\mathrm{NHR}^{2}\right)_{3} \\
\text { (11) non-gem }-\mathrm{N}_{3} \mathrm{P}_{3} \mathrm{Cl}_{3}\left(\mathrm{NHR}^{2}\right)_{3}{ }^{k}\end{array}$ & & & $j$ & & \\
\hline $\begin{array}{l}\text { (11) non-gem- } \mathrm{N}_{3} \mathrm{P}_{3} \mathrm{Cl}_{3}\left(\mathrm{NHR}^{2}\right)_{3}{ }_{\text {cis- }(11)^{i}} \\
\end{array}$ & & & 16.0 & & \\
\hline $\operatorname{trans}-(11)^{i}$ & & & 15.8 & & \\
\hline (12) $\mathrm{N}_{3} \mathrm{P}_{3} \mathrm{Cl}_{5}\left(\mathrm{NHR}^{3}\right)$ & 78 & 21.3 & 13.0 & & 47.6 \\
\hline (13) gem- $-\mathrm{N}_{3} \mathrm{P}_{3} \mathrm{Cl}_{4}\left(\mathrm{NHR}^{3}\right)_{2}$ & 111 & 21.3 & & -1.0 & 46.2 \\
\hline (14) non-gem- $\mathrm{N}_{3} \mathrm{P}_{3} \mathrm{Cl}_{4}\left(\mathrm{NHR}^{3}\right)_{2}$ & $128-136$ & & & & \\
\hline $\begin{array}{l}\left.\operatorname{cis}^{-(14}\right)^{i} \\
\left.\operatorname{trans}^{-(14}\right)^{i}\end{array}$ & & 22.7 & 15.2 & & 51.6 \\
\hline trans $-(14)^{i}$ & & 22.2 & 15.0 & & 47.1 \\
\hline $\begin{array}{l}\text { (15) gem- } \mathrm{N}_{3} \mathrm{P}_{3} \mathrm{Cl}_{3}\left(\mathrm{NHR}^{3}\right)_{3} \\
\text { (16) non-gem- } \mathrm{N}_{3} \mathrm{P}_{3} \mathrm{Cl}_{3}\left(\mathrm{NHR}^{3}\right)_{3}\end{array}$ & & $22.8^{m}$ & $17.1^{m}$ & $1.5^{\mathrm{m}}$ & \\
\hline 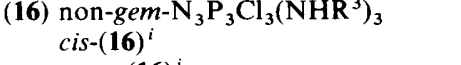 & $165-168$ & & 17.3 & & \\
\hline $\operatorname{trans}-(16)^{i}$ & & & $\begin{array}{l}18.0(1) \\
17.2(2)^{n}\end{array}$ & & 43.6 \\
\hline (17) $\mathrm{gem}-\mathrm{N}_{3} \mathrm{P}_{3} \mathrm{Cl}_{2}\left(\mathrm{NHR}^{3}\right)_{4}$ & $106-109$ & 23.6 & & 2.1 & 50.8 \\
\hline (18) $\mathrm{N}_{3} \mathrm{P}_{3} \mathrm{Cl}\left(\mathrm{NHR}^{3}\right)_{5}$ & $53-56^{\circ}$ & & & & \\
\hline (19) $\mathrm{N}_{3} \mathrm{P}_{3}\left(\mathrm{NHR}^{3}\right)_{6}$ & 221 & & & 6.4 & \\
\hline (20) $\mathrm{N}_{3} \mathrm{P}_{3}\left(\mathrm{NHR}^{2}\right)\left(\mathrm{NMe}_{2}\right)_{5}$ & 119 & 25.3 & 15.0 & & 42.3 \\
\hline $\begin{array}{l}\text { (21) gem }-\mathrm{N}_{3} \mathrm{P}_{3}\left(\mathrm{NHR}^{2}\right)_{2}\left(\mathrm{NMe}_{2}\right)_{4} \\
\text { (22) non-gem }-\mathrm{N}_{3} \mathrm{P}_{3}\left(\mathrm{NHR}^{2}\right)_{2}\left(\mathrm{NMe}_{2}\right)_{4}\end{array}$ & 155 & 25.9 & & 5.6 & 44.5 \\
\hline 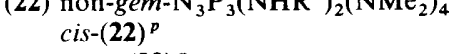 & $47-50$ & 26.2 & 15.3 & & 43.6 \\
\hline trans $-(22)^{p}$ & & 26.8 & 15.9 & & 40.6 \\
\hline (23) $\mathrm{N}_{3} \mathrm{P}_{3}\left(\mathrm{NHR}^{3}\right)\left(\mathrm{NMe}_{2}\right)_{5}$ & 130 & 26.3 & 16.4 & & 42.2 \\
\hline (24) non-gem- $\mathrm{N}_{3} \mathrm{P}_{3}\left(\mathrm{NHR}^{3}\right)_{2}\left(\mathrm{NMe}_{2}\right)_{4}$ & $134-141$ & 26.3 & 16.3 & & 43.6 \\
\hline (25) gem- $\mathrm{N}_{3} \mathrm{P}_{3}\left(\mathrm{NHR}^{3}\right)_{4}\left(\mathrm{NMe}_{2}\right)_{2}{ }^{q}$ & $149-151$ & & & & \\
\hline (26) $\mathrm{N}_{3} \mathrm{P}_{3}\left(\mathrm{NHR}^{1}\right)(\mathrm{OMe})_{5}$ & 127 & 19.8 & 16.7 & & 74.5 \\
\hline (27) gem- $\mathrm{N}_{3} \mathrm{P}_{3}\left(\mathrm{NHR}^{1}\right)_{2}(\mathrm{OMe})_{4}$ & 139 & 18.0 & & 7.0 & 66.3 \\
\hline $\begin{array}{l}\text { (28) non-gem- }-\mathrm{N}_{3} \mathrm{P}_{3}\left(\mathrm{NHR}^{1}\right)_{2}(\mathrm{OMe})_{4} \\
\text { cis-(28) }\end{array}$ & $125-142$ & & & & \\
\hline $\begin{array}{l}\operatorname{cis}-(\mathbf{2 8})^{p} \\
\text { trans }(\mathbf{2 8})^{p}\end{array}$ & $153-155$ & $\begin{array}{l}18.1 \\
18.7\end{array}$ & $\begin{array}{l}15.8 \\
15.6\end{array}$ & & $\begin{array}{l}6.6 \\
71.7\end{array}$ \\
\hline $\begin{array}{l}\text { trans }-(28)^{p} \\
\text { (29) } \mathrm{N}_{3} \mathrm{P}_{3}\left(\mathrm{NHR}^{2}\right)(\mathrm{OMe})_{5}\end{array}$ & 103 & 19.9 & 16.9 & & 78.3 \\
\hline (30) $\mathrm{gem}-\mathrm{N}_{3} \mathrm{P}_{3}\left(\mathrm{NHR}^{2}\right)_{2}(\mathrm{OMe})_{4}$ & 154 & 18.9 & & 6.8 & 65.9 \\
\hline (31) non-gem- $\mathrm{N}_{3} \mathrm{P}_{3}\left(\mathrm{NHR}^{2}\right)_{2}(\mathrm{OMe})_{4}$ & $107-167$ & & & & \\
\hline$c i s-(\mathbf{3 1})^{p}$ & & 17.9 & 15.1 & & 69.9 \\
\hline trans $-(\mathbf{3 1})^{p}$ & & 17.9 & 15.0 & & 70.3 \\
\hline (32) $\mathrm{N}_{3} \mathrm{P}_{3}\left(\mathrm{NHR}^{3}\right)(\mathrm{OMe})_{5}$ & 76 & 19.1 & 16.5 & & 71.4 \\
\hline (33) gem- $-\mathrm{N}_{3} \mathrm{P}_{3}\left(\mathrm{NHR}^{3}\right)_{2}(\mathrm{OMe})_{4}$ & 66 & 18.7 & & 7.7 & 66.0 \\
\hline $\begin{array}{l}\text { (34) non-gem- } \mathrm{N}_{3} \mathrm{P}_{3}\left(\mathrm{NHR}^{3}\right)_{2}(\mathrm{OMe})_{4} \\
\text { cis-(34) }\end{array}$ & Viscous oil & 18.4 & & & \\
\hline trans- $(34)^{p}$ & & 18.4 & 16.0 & & 68.3 \\
\hline (35) gem $-\mathrm{N}_{3} \mathrm{P}_{3}\left(\mathrm{NHR}^{3}\right)_{3}(\mathrm{OMe})_{3}{ }^{r}$ & Viscous oil & & & & \\
\hline (36) non-gem- $\mathrm{N}_{3} \mathrm{P}_{3}\left(\mathrm{NHR}^{3}\right)_{3}(\mathrm{OMe})_{3}$ & Viscous oil & & 15.0 & & \\
\hline (37) gem $-\mathrm{N}_{3} \mathrm{P}_{3}\left(\mathrm{NHR}^{3}\right)_{4}(\mathrm{OMe})_{2}$ & $112-117$ & 17.5 & & 6.8 & 59.2 \\
\hline
\end{tabular}

${ }^{a}$ Chemical shifts ( $\delta$ /p.p.m.) are with reference to external $85 \% \mathrm{H}_{3} \mathrm{PO}_{4}$; upfield shifts negative. ${ }^{b} \mathrm{R}^{1}=\mathrm{Ph}, \mathrm{R}^{2}=\mathrm{C}_{6} \mathrm{H}_{4} \mathrm{Me}-p$, and $\mathrm{R}^{3}=\mathrm{C}_{6} \mathrm{H}_{4}(\mathrm{OMe})$ p. ${ }^{c} \mathrm{P}^{1}=\equiv P C l_{2}, \equiv P\left(\mathrm{NMe}_{2}\right)_{2}$, or $\equiv P(\mathrm{OMe})_{2} .{ }^{d} \mathrm{P}^{2}=\equiv P C l(\mathrm{NHR}), \equiv P(\mathrm{NHR})\left(\mathrm{NMe}_{2}\right)$, or $P(\mathrm{NHR})(\mathrm{OMe}) .{ }^{e} \mathrm{P}^{3}=\equiv P(\mathrm{NHR})_{2} .{ }^{f} \mathrm{Lit}$. m.p. same $(\mathrm{ref}$. 3). ${ }^{g}$ Lit. m.p. $207.5-208.5^{4}$ and $212^{\circ} \mathrm{C}^{3{ }^{h}}{ }^{h}$ Lit. m.p. $165^{\circ} \mathrm{C}$; no spectral data reported. ${ }^{3}{ }^{i}$ Assignment for individual isomers is based on the relative intensities and the known isomeric composition from the ${ }^{1} \mathrm{H}$ n.m.r. spectrum of the methoxylated derivative. ${ }^{j}$ Not isolated; the $\equiv P C l_{2}, \equiv P C l(N H R)$ and $\equiv P(\mathrm{NHR})_{2}$ signals are detected in the regions $\delta 23.0,14.2$, and -1.0 respectively. ${ }^{k}$ The ${ }^{31} \mathrm{P}$ n.m.r. spectrum of the reaction mixture obtained from a 1:4 $\mathrm{N}_{3} \mathrm{P}_{3} \mathrm{Cl}_{6}: \mathrm{NH}_{2} \mathrm{R}$ stoicheiometric reaction in $\mathrm{MeCN}$ shows two additional lines apart from the intense signals arising from the nongeminal bis(amino) derivatives. These are ascribed to the cis and trans non-geminal tris(amino) derivatives on the basis of trends in phosphorus chemical shifts established for (amino)chlorocyclotriphosphazenes (see ref. 7). ${ }^{\prime}$ Lit. m.p. same (Y. Kobayashi, L. A. Chasin, and L. B. Clapp, Inorg. Chem., 1963, 2, 212). ${ }^{m}$ Broad peaks in the specified region. ${ }^{n}$ Data from 109.3-MHz spectrum; values in parentheses represent intensity ratios. ${ }^{\circ}$ Contains equal amounts of (17), (18), and (19). ${ }^{p}$ Assignments for individual isomers are based on the relative intensities and the known isomeric composition from the ${ }^{1} \mathrm{H}$ n.m.r. spectra. ${ }^{9}$ Relative intensities of $\mathrm{P}-\mathrm{N} M e_{2}$ and $\mathrm{P}-\mathrm{NHC}_{6} \mathrm{H}_{4}(\mathrm{OMe})$-p protons in the ${ }^{1} \mathrm{H}$ n.m.r. spectrum are used to establish the molecular formula; ${ }^{31} \mathrm{P}$ n.m.r. spectrum could not be recorded because of the tiny amount of the sample. ${ }^{r}$ Obtained only as a mixture with (36); ${ }^{31} \mathrm{P}$ n.m.r. spectrum was not recorded. 
triethylamine is used as the hydrogen halide acceptor instead of the reacting amine itself in the above solvents, the geminal bis(amino) product is formed exclusively. The structures of the products formed in these reactions were elucidated by ${ }^{31} \mathrm{P}$ n.m.r. spectroscopy. The data are summarized in Table 2 . In some instances the distribution of products has also been determined

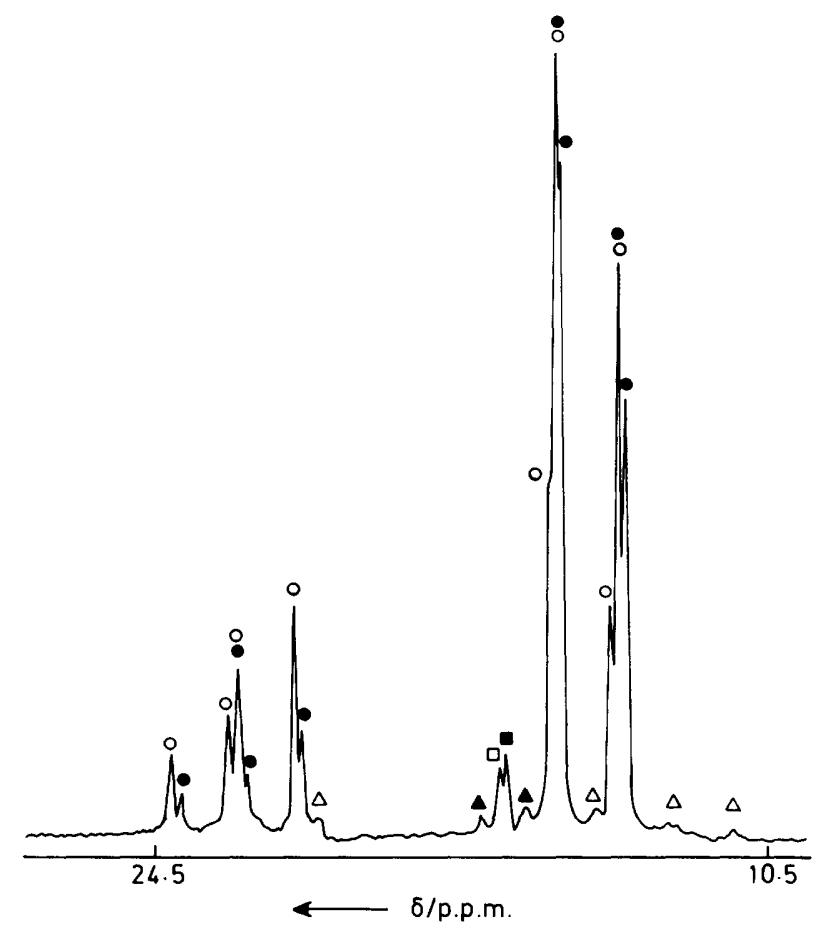

Figure 1. The ${ }^{31} \mathrm{P}-\left\{{ }^{1} \mathrm{H}\right\}$ n.m.r. spectrum $\left(32.2 \mathrm{MHz}, \mathrm{CDCl}_{3}\right)$ of the reaction mixture obtained by treatment of $\mathrm{N}_{3} \mathrm{P}_{3} \mathrm{Cl}_{6}$ with $p$-toluidine (1:4 stoicheiometry) in $\mathrm{MeCN}$ at $25^{\circ} \mathrm{C}$. In addition to the signals shown, weak signals were observed at $\delta-0.5$ to -2.0 due to geminal bis- and tris-(amino) derivatives. $\bigcirc=$ cis-(9), $\mathbf{O}=$ trans-(9), $\square=$ cis(11), $\mathbf{\square}=$ trans $-(11), \Delta=(7)$, and $\Delta=(10)$
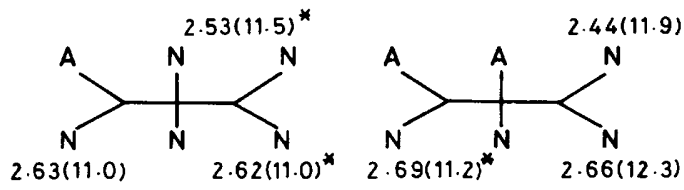

(23)

$$
\text { cis-(24) }
$$

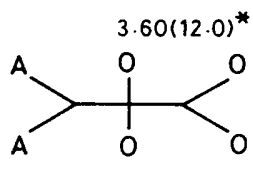

(33)

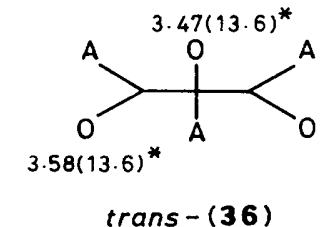

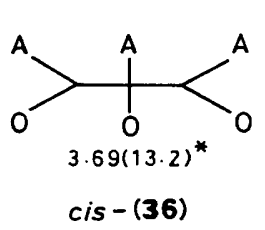

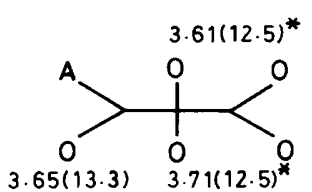

(32)

cis $-(36)$ trans $-(36)$ from the ${ }^{31} \mathrm{P}$ n.m.r. spectrum of the reaction mixture. A typical spectrum is illustrated in Figure 1. An $\mathrm{AB}_{2}$ pattern is consistent with a non-geminal structure whereas for the geminal derivative, an $\mathrm{AX}_{2}$ pattern is observed. The $\equiv P(\mathrm{NHR})_{2}(\mathrm{R}=$ aryl) signal appears at a higher field compared to the $\equiv P C l(N H R)$ or $\equiv \mathrm{PCl}_{2}$ signals. With increasing degree of chlorine replacement, the chemical shifts of the $\equiv P(\mathrm{NHR})_{2}, \equiv P C \mathrm{Cl}(\mathrm{NHR})$, and $\equiv P \mathrm{Pl}_{2}$ signals move downfield. Similar trends have been observed for dimethylamino-, piperidino-, ${ }^{7}$ aziridino-,${ }^{8}$ and dimethylamino(triphenylphosphazenyl)-cyclophosphazene ${ }^{9}$ derivatives. The $\equiv P C l(N H R)$ signal lies upfield to those of $\equiv P \mathrm{Pl}_{2}$ as observed for (primary amino)cyclotriphosphazenes. The phosphorus chemical shifts for the cis isomers lie slightly downfield to those of the trans isomers except for (22). The available data in the literature for pairs of geometrical isomers are limited ${ }^{7}$ and it is not clear whether the trend observed in the present study is of general validity.

It is difficult to ascertain the isomeric configurations of the (arylamino)chlorocyclotriphosphazenes from ${ }^{31} \mathrm{P}$ n.m.r. spectroscopy. For this purpose, ${ }^{1} \mathrm{H}$ n.m.r. spectra of the corresponding dimethylamino and methoxy derivatives are used. The ratio of geometrical isomers changes during dimethylaminolysis since the amine hydrochloride formed acts as an inverting agent; ${ }^{2,10}$ during methoxylation such a change does not occur. It must be stressed that in these systems we have found no evidence $\left({ }^{1} \mathrm{H}\right.$ and ${ }^{31} \mathrm{P}$ n.m.r.) for a geminal-nongeminal transformation of the type recently observed in the reaction of gem- $\mathrm{N}_{3} \mathrm{P}_{3} \mathrm{Cl}_{4}\left(\mathrm{NH}_{2}\right)_{2}$ with sodium n-propoxide. ${ }^{11}$ The proton n.m.r. data for dimethylamino and methoxy derivatives of ( $p$-anisidino)cyclotriphosphazenes are summarized in Figure 2; for the corresponding anilino and $p$-toluidino derivatives similar trends are observed both in the chemical shifts (deviation $\pm 0.08 \delta$ unit) and coupling constants (deviation $\pm 1 \mathrm{~Hz}$ ). The assignment of the chemical shifts to protons in different environments can be made unambiguously for compounds (21), trans-(22), trans-(24), (25), (27), trans-(28), (30), trans-(31), (33), and trans-(34) from a consideration of the relative intensities and also on the basis of the presence or absence of 'virtual coupling'. 5 The assignments for other compounds are made on the basis of the shielding effect of the

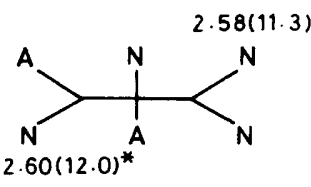

trans $-(\mathbf{2 4})$

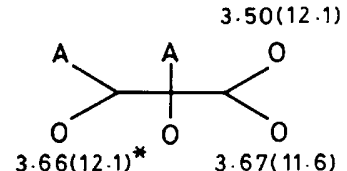

cis $-(34)$

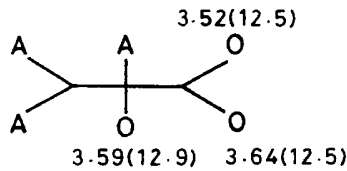

(35)

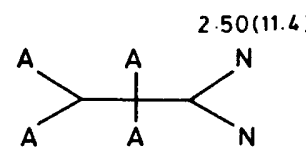

(25)

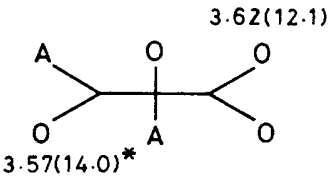

.rans $-(34)$



(37)

Figure 2. Hydrogen-1 n.m.r. data for dimethylamino and methoxy derivatives of ( $p$-anisidino)cyclotriphosphazenes. The values shown represent proton chemical shifts with ${ }^{3} J(\mathrm{P}-\mathrm{H})$ (in $\mathrm{Hz}$ ) in parentheses. $\mathrm{A}=\mathrm{NHC}_{6} \mathrm{H}_{4}(\mathrm{OMe})-p, \mathrm{~N}=\mathrm{NMe}_{2}, \mathrm{O}=\mathrm{OMe}$, and ${ }^{*}=$ virtual coupling 


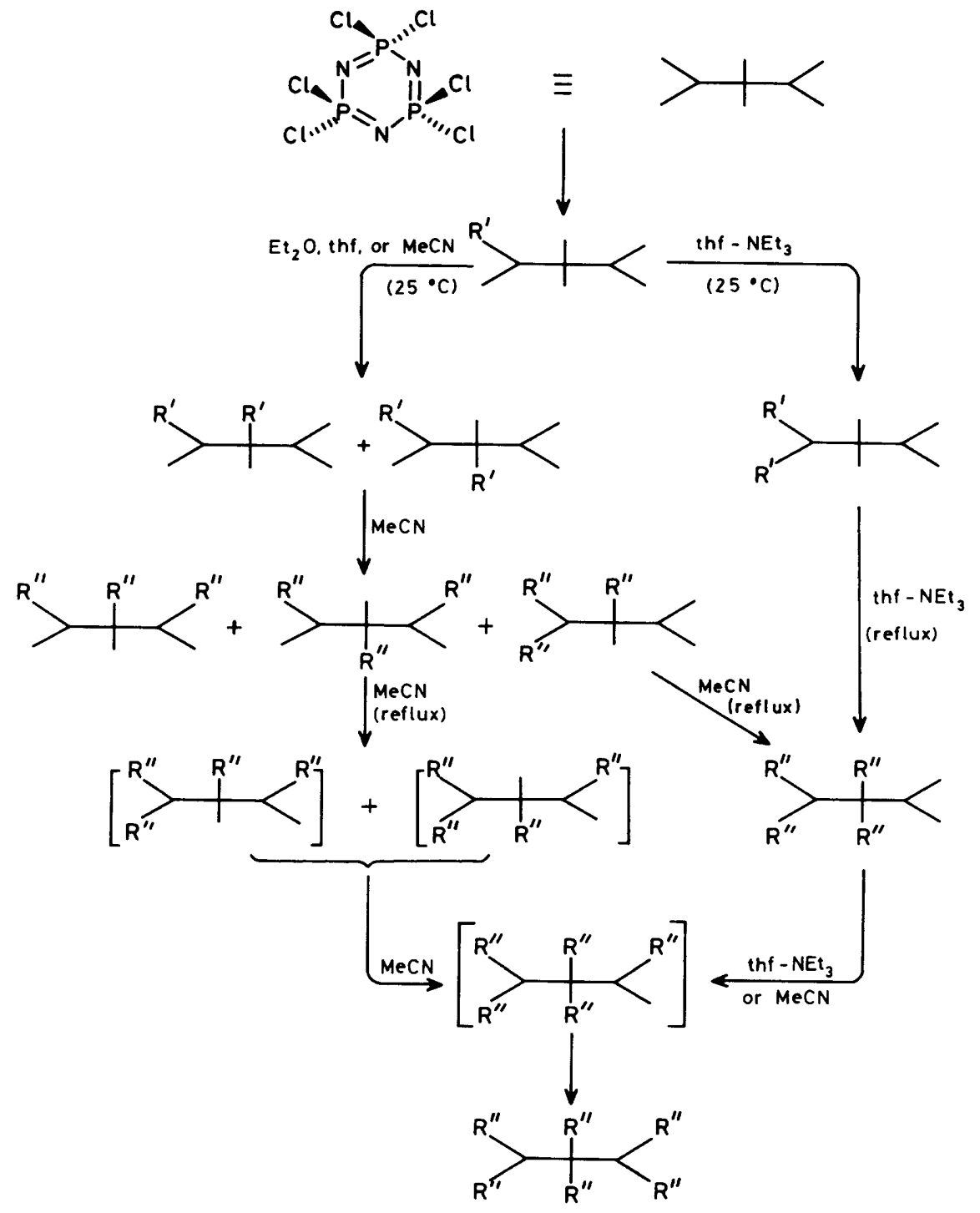

Figure 3. Chlorine replacement pattern established for the reactions of $\mathrm{N}_{3} \mathrm{P}_{3} \mathrm{Cl}_{6}(1)$ with aromatic primary amines (square brackets indicate reactive intermediates under appropriate reaction conditions). $\mathrm{R}^{\prime}=\mathrm{NHPh}, \mathrm{NHC}_{6} \mathrm{H}_{4} \mathrm{Me}-p$, or $\mathrm{NHC}_{6} \mathrm{H}_{4}(\mathrm{OMe})-p ; \mathrm{R}^{\prime \prime}=\mathrm{NHC}_{6} \mathrm{H}_{4}(\mathrm{OMe})-p$ only

arylamino groups on the protons of the substituent present in a cis orientation. It appears that this shielding effect of an arylamino group is less than that of a phenyl, ${ }^{12,13}$ phenoxy, ${ }^{14}$ or $p$-cresoxy ${ }^{15}$ substituent.

The P-N $H$ signal appears as a doublet $\left[{ }^{2} J(\mathrm{PNH})=10-12\right.$ $\mathrm{Hz}]$ or as a broad peak; the chemical shift is in the range $\delta 4.5-$ 6.0. After dimethylaminolysis or methoxylation, the $\mathrm{P}-\mathrm{N} H$ signal generally moves upfield, in some cases by as much as 1.0 $\delta$ unit; the coupling constant $\left[{ }^{2} J(\mathrm{PNH})\right]$ is slightly decreased $(8-9 \mathrm{~Hz})$. The $\mathrm{NH}$ protons of geminal bis(arylamino) derivatives are more shielded than those of the non-geminal derivatives. This observation is in line with the results obtained for (primary amino)cyclotriphosphazenes. ${ }^{16}$ This trend is not always true for dimethylamino or methoxy derivatives of (arylamino)cyclotriphosphazenes.

In the ${ }^{31} \mathrm{P}$ n.m.r. spectra of the methoxy derivatives (27), (30), (33), and (37), the $\equiv P(\mathrm{NHR})_{2}$ signal is considerably deshielded compared to the chloro or dimethylamino derivatives; ${ }^{2} J(\mathrm{PNP})$ increases with the increasing number of methoxy substituents (Table 2).

Chlorine Replacement Pattern.-The reactions of (1) with several primary amines have been reported and these studies have been largely carried out in solvents of low polarity such as diethyl ether, chloroform, or aromatic hydrocarbons. ${ }^{5}$ Ethylamine gives predominantly non-geminal bis(amino) derivatives ${ }^{16}$ whereas t-butylamine gives exclusively geminal products; ${ }^{17}$ isopropylamine behaves in an intermediate fashion. ${ }^{16,18} \mathrm{~A}$ geminal substitution pattern is observed for the reaction of (1) with aniline in benzene. ${ }^{3.4}$

The chlorine replacement patterns established in the present study for the reaction of (1) with aniline, $p$-toluidine, and $p$ anisidine are illustrated in Figure 3. Non-geminal bis(arylamino) derivatives are formed predominantly in $\mathrm{MeCN}$; even in diethyl ether and thf at ambient temperature, a non-geminal bis(arylamino) derivative is the predominant product. When triethylamine is used as a hydrogen halide acceptor, a geminal bis(arylamino) derivative is formed exclusively in all of the above solvents. Thus, the nature of the solvent and the use of a tertiary amine as a hydrogen halide acceptor play an important role in determining the chlorine replacement pattern in the reaction of (1) with aromatic primary amines.

The formation of non-geminal bis(amino) products [(9) and (14)] occur by an $S_{\mathrm{N}} 2(\mathrm{P})$ mechanism and that of geminal products $\left[(\mathbf{8})\right.$ and (13)] by a base-catalysed $E_{1}$ (c.b.) (c.b. = conjugate base) mechanism from their respective 
mono(arylamino) derivatives. Evidence for these mechanisms has been obtained from kinetic studies and a detailed discussion of the mechanistic aspects is presented in the following paper. ${ }^{2}$

Replacement of chlorine atoms beyond the bis stage has been investigated only for $p$-anisidine because of its greater reactivity. At the tris stage of chlorine replacement, geminal and nongeminal products [(15) and (16)] are formed in comparable amounts in MeCN. The formation of non-geminal and geminal tris derivatives in $\mathrm{MeCN}$ can be readily explained on the basis of a competition between $S_{\mathrm{N}} 2(\mathrm{P})$ and $E_{1}$ (c.b.) mechanisms. Only in the reaction of (1) with ethylamine, has a non-geminal tris(primary amino) derivative been isolated previously. ${ }^{16}$ The tris derivatives could not be identified by t.l.c. or by ${ }^{31} \mathrm{P}$ n.m.r. spectroscopy in the reactions of (1) with $p$-anisidine in thf in the presence of triethylamine. Under the experimental conditions, the $\equiv \mathrm{PCl}(\mathrm{NHR})$ centre is more prone to undergo substitution by an $E_{1}$ (c.b.) mechanism. The reaction of (16) with two molar equivalents of $p$-anisidine in MeCN yields (19) and the unreacted starting material (16). The rapid removal of all the three chlorine atoms is certainly due to a fast dissociative pathway involving the heterolysis of the $\mathrm{P}-\mathrm{Cl}$ bond either by an $S_{\mathrm{N}} 1(\mathrm{P})^{19}$ or $E_{1}$ (c.b.) mechanism. ${ }^{2}$

The geminal tetrakis(arylamino) derivative (17) is the sole product at the tetrakis stage of chlorine replacement irrespective of the reaction media and experimental conditions for the reaction of (1) with $p$-anisidine. Similar results have been obtained for the reactions of $(\mathbf{1})$ with aliphatic primary amines. ${ }^{5}$

\section{Conclusions}

It has been generally believed for quite some time that the chlorine replacement pattern in the aminolysis reaction of chlorocyclophosphazenes is determined only by the attacking nucleophile and is not dependent on the substituent already present on the phosphazene ring. ${ }^{20.21}$ Recent studies have shown that the above conclusion is too simplistic in its general validity. $9,19,22,23$ The halogen replacement pattern is determined by the combined effects of the attacking nucleophile, the substituent present on the phosphazene ring, the leaving group, and to some extent the nature of the solvent. The systematic investigations carried out in the present study with three aromatic primary amines further highlight the importance of all the above factors and also provide a firm basis for characterization of the products of the reactions chosen for kinetic studies. $^{2}$

\section{Acknowledgements}

We thank the Indian Space Research Organization (Trivandrum) for support, Professor A. R. Vasudeva Murthy for his kind encouragement, and Drs. R. Keat and M. Woods for ${ }^{31} \mathbf{P}$ n.m.r. spectra ( 40.5 or $36.4 \mathrm{MHz}$ ). Thanks are also due to $\mathrm{Mr}$. B. Raju, Molecular Biophysics Unit of this Institute, for ${ }^{31} \mathrm{P}$ n.m.r. measurements at $32.2 \mathrm{MHz}$ and Dr. K. Nagarajan, Ciba Research Centre (Bombay), for elemental analyses.

\section{References}

1 Part 29, K. C. Kumara Swamy, S. S. Krishnamurthy, A. R. Vasudeva Murthy, R. A. Shaw, and M. Woods, Indian J. Chem., Sect. A, in the press.

2 S. Ganapathiappan and S. S. Krishnamurthy, following paper.

3 V. B. Desai, R. A. Shaw, and B. C. Smith, J. Chem. Soc. A, 1970, 2023

4 H. Lederle, G. Ottman, and E. Kober, Inorg. Chem., 1966, 5, 1818.

5 S. S. Krishnamurthy, A. C. Sau, and M. Woods, Adv. Inorg. Chem. Radiochem., 1978, 21, 41.

6 K. C. Kumara Swamy and S. S. Krishnamurthy, Indian J. Chem. Sect. A, 1984, 23, 717 .

7 R. Keat, R. A. Shaw, and M. Woods, J. Chem. Soc., Dalton Trans., 1976,1582

8 A. A. Van der Huizen, A. P. Jekel, J. Rusch, and J. C. Van de Grampel, Recl. Trav. Chim. Pays-Bas., 1981, 100, 343.

9 S. S. Krishnamurthy, P. Ramabrahmam, A. R. Vasudeva Murthy, R. A. Shaw, and M. Woods, Z. Anorg. Allg. Chem., 1985, 522, 226.

10 R. Keat and R. A. Shaw, J. Chem. Soc., 1965, 4067.

11 J. K. Fincham, M. B. Hursthouse, H. G. Parkes, L. S. Shaw, and R. A. Shaw, J. Chem. Soc., Chem. Commun., 1985, 252.

12 S. Das, R. A. Shaw, and B. C. Smith, J. Chem. Soc., Dalton Trans., 1973, 1883.

13 B. Grushkin, A. J. Berlin, J. L. McClanahan, and R. G. Rice, Inorg. Chem., 1966, 5, 172; B. Grushkin, M. G. Sanchez, M. V. Ernest, J. L. McClanahan, G. E. Ashby, and R. G. Rice, ibid., 1965, 4, 1538.

14 D. Dell, B. W. Fitzsimmons, R. Keat, and R. A. Shaw, J. Chem. Soc. A, 1966, 1680.

15 S. Karthikeyan and S. S. Krishnamurthy, Z. Anorg. Allg. Chem., 1984, 513, 231.

16 R. N. Das, R. A. Shaw, B. C. Smith, and M. Woods, J. Chem. Soc., Dalton Trans., 1973, 709.

17 S. K. Das, R. Keat, R. A. Shaw, and B. C. Smith, J. Chem. Soc., 1965 , 5032.

18 D. J. Lingley, R. A. Shaw, M. Woods, and S. S. Krishnamurthy, Phosphorus Sulfur, 1978, 4, 379.

19 K. V. Katti and S. S. Krishnamurthy, J. Chem. Soc, Dalton Trans., $1985,285$.

20 R. Keat and R. A. Shaw, Angew. Chem., Int. Ed. Engl., 1968, 7, 212.

21 V. B. Desai, R. A. Shaw, and B. C. Smith, J. Chem. Soc. A, 1969, 1977.

22 K. C. Kumara Swamy, M. D. Poojary, S. S. Krishnamurthy, and H. Manohar, J. Chem. Soc., Dalton Trans., 1985, 1881.

23 K. C. Kumara Swamy and S. S. Krishnamurthy, Phosphorus Sulfur, 1983, 18, 241; Inorg. Chem., 1986, 25, 920.

Received 6th December 1985; Paper 5/2141 